\title{
Propiedades psicométricas del Test de Motivaciones Existenciales en adultos jóvenes
}

\author{
Aída Mercado Maya \\ Érika Robles Estrada \\ Johannes Oudhof van Barneveld \\ Universidad Autónoma del \\ Estado de México, Ciudad de México
}

\begin{abstract}
Recibido: 21 de marzo del 2019 / Aceptado: 20 de abril del 2019 doi: 10.26439/persona2019.n022(1).4090
\end{abstract}

El objetivo de este estudio fue obtener las propiedades psicométricas del Test de Motivaciones Existenciales de Längle y Eckhardt en una muestra de adultos jóvenes, residentes de la zona metropolitana del Valle de Toluca, México. El instrumento fue aplicado a 300 participantes. A partir de los análisis estadísticos efectuados, se puede establecer que el test es adecuado para la medición de las motivaciones existenciales en adultos jóvenes, de acuerdo con las evidencias de confiabilidad y validez obtenidas.

motivaciones existenciales / validez / confiabilidad / adultos jóvenes

\section{Psychometric Properties of the Test of Existential Motivations in Young Adults}

The purpose of this study was to obtain the psychometric properties of the Test of Existential Motivations created by Längle \& Eckhard in a sample of young adults living in the metropolitan area of the Valle de Toluca, Mexico. The instrument was assessed in 300 subjects. Based on the statistical analysis, it can be concluded that the test is adequate to measure existential motivations in young adults, considering the evidence of reliability and validity.

existential motivations / validity / reliability / young adults

Correo electrónico: aidamercadom@hotmail.com 


\section{INTRODUCCIÓN}

La juventud o vida adulta temprana, desde la psicología del desarrollo, tiene lugar entre los 18 y los 25 años. Como etapa del desarrollo, implicaba hace un par de décadas la resolución de aspectos tales como alcanzar la intimidad, elegir una carrera y el logro del éxito vocacional. Berger (2009) refiere que dicha etapa se caracteriza por más años de educación, matrimonios tardíos, menos nacimientos y postergación de la elección de la profesión.

La Organización de las Naciones Unidas (ONU, 2016) plantea que "los jóvenes representan el conjunto de la población de entre 15 y 24 años de edad, es decir, el $18 \%$ del total de la población mundial" (párr. 1). En tanto que la Comisión Económica para América Latina y el Caribe, que también pertenece a la ONU, establece que la juventud se acota al grupo de edad de entre $15 \mathrm{y}$ 29 años (Trucco y Ullman, 2015).

En México, los datos de la encuesta intercensal (2016), efectuada por el Instituto Nacional de Estadística y Geografía, indican que el monto de la población joven de 15 a 29 años ha ascendido a 30,6 millones. Esto representa $25,7 \%$ de la población nacional, de la cual 50,9 \% son mujeres y $49,1 \%$ son hombres. En cuanto a su estructura por edad, $35,1 \%$ tiene entre 15 y 19 años; $34,8 \%$, entre 20 y 24 años; y 30,1 \%, entre 25 y 29 años. El 46,5\% de los jóvenes de 25 a 29 años de edad cuentan con secundaria o un nivel de escolaridad menor. En tanto que el 66,8 \% de los jóvenes de 15 a 29 años no asisten a la escuela. En jóvenes de 20 a 24 años, el porcentaje con educación superior es de $27,5 \%$, y para el grupo de 25 a 29 años es de $27,8 \%$. Sin embargo, destaca que, para este último grupo de edad, el $46,5 \%$ cuenta con secundaria o un grado de escolaridad menor, debido a que a esta edad la mayor parte de la población ha concluido su instrucción formal.

Los resultados correspondientes al primer trimestre de 2018 de la encuesta nacional de ocupación y empleo, realizada por el Instituto Nacional de Estadística y Geografía, muestran que la tasa de desocupación de la PEA de 15 años a más es de $3,2 \%$ en el ámbito nacional; es decir, 1,7 millones de personas, de las cuales $60,8 \%$ son hombres y $39,2 \%$ son mujeres. Por otro lado, el 5,8\% de los jóvenes de 15 a 29 años están desocupados. La tasa más alta de desocupación en la población joven se presenta en el grupo de 20 a 24 años con $6,7 \%$; la tasa es $6,4 \%$ para el grupo de 15 a 19 años y 4,6 \% para el grupo de 25 a 29 años.

Los datos documentados por dicha encuesta reflejan una alta proporción de ocupaciones informales que absorben a esta población activa. Los resultados señalan que de las 15 millones de personas jóvenes ocupadas de 15 a 29 años, 59,5\% (poco más de 8,9 millones) labora en el sector informal. El trabajo informal en la población joven es predominantemente masculino. Así, 6 de cada 10 personas ocupadas en trabajos informales $(65,8 \%)$ son hombres, en tanto que $34,2 \%$ son mujeres. Por grupos de edad, el trabajo informal predomina en la población más joven: $28,8 \%$ de las personas que se desempeñan en actividades informales tiene de 15 a 19 años, mientras que solo 8 de cada 100 personas que se ocupan en el 
sector formal de la economía están en dicho rango. La vulnerabilidad de la población que se desempeña en un empleo informal se manifiesta de varias maneras. Una de ellas es la carencia de prestaciones laborales: 8 de cada 10 personas jóvenes de 15 a 29 años $(85,3 \%)$ no goza de este tipo de beneficios, en tanto que $13,7 \%$ disfruta de prestaciones laborales con exclusión del acceso a servicios de salud. El nivel de ingresos percibidos por su trabajo es otra arista en la que se manifiesta la vulnerabilidad del trabajo informal. En ese sentido, la proporción de jóvenes de 15 a 29 años que trabaja de manera informal y no recibe remuneración es de 13,7 \%. Por su parte, de los 8,9 millones de personas jóvenes con ocupación informal, el $78 \%$ percibe ingresos por su trabajo; de ellas, 30,8\% recibe hasta 1 salario mínimo, casi 1 de cada 2 (46,6\%) recibe más de 1 y hasta 2 salarios mínimos, $15,1 \%$ recibe más de 2 y hasta 3 salarios mínimos, y solo $7,4 \%$ percibe 3 o más salarios mínimos. Por sexo, la diferencia es más evidente, ya que en lo que respecta a las mujeres de 15 a 29 años, 42,5\% percibe menos de 1 salario mínimo, mientras que $24,8 \%$ de los hombres jóvenes perciben este nivel de ingreso mensual; asimismo $9 \%$ de los jóvenes que trabajan de manera informal recibe más de 3 salarios mínimos, proporción que en las mujeres jóvenes es de solo 4,4\%. Por otra parte, el monto de la población joven no económicamente activa es de 15 millones de jóvenes de 15 a 29 años. Este conjunto de población se caracteriza por ser en su mayoría mujeres: $65,2 \%$ por $34,8 \%$ hombres. De las mujeres jóvenes no activas económicamente, $61,5 \%$ asiste a la escuela y 1 de cada $2(54,4 \%)$ tiene entre
15 y 19 años. Lo anterior significa que de las 15 millones de personas jóvenes de 15 a 29 años que solo realizan actividades no económicas, 9,2 millones se mantiene dentro del sistema educativo nacional y 5,8 millones no asiste a la escuela. De las personas jóvenes no económicamente activas y que además no asisten a la escuela, sobresale que $86,5 \%$ (5 millones) son mujeres, en tanto que los hombres representan el 13,5\%. La mayor proporción de mujeres económicamente no activas y que no asisten a la escuela se concentra en el grupo de 25 a 29 años, 41,3 \%; mientras que en los hombres se concentra en el grupo más joven, 43,5\% tiene de 15 a 19 años.

Los datos referidos revelan las circunstancias que colocan a este sector de la población en una posición no favorable y de vulnerabilidad -lo cual es más desalentador en el caso de las mujeres- en lo que respecta a la construcción de un futuro prometedor en el que puedan desarrollar sus talentos, capacidades y potencialidades, de manera que cuenten con los recursos que les permitan acceder a una mejor calidad de vida y bienestar psicológico. Aunado a lo anterior, se tiene que en los llamados países industrializados los jóvenes postergan los roles adultos tanto tiempo como les sea posible. Por ello, la juventud ha adquirido un nuevo matiz en el ciclo del desarrollo humano, en el que se encuentran implicadas cuatro dimensiones básicas (física, cognitiva, emocional y social) interdependientes, es decir, que se complementan unas con otras. Así, las capacidades cognoscitivas pueden depender de la salud física y emocional, al igual que de la experiencia social; y el desarrollo social se encuentra influido por la maduración 
biológica, la comprensión cognoscitiva y las reacciones emocionales (Rice, 2006).

La evolución del ser humano tiene lugar en un ciclo natural de desarrollo que implica cambios, adaptaciones, transformaciones y dificultades inherentes a cada etapa de la vida. Por ello, Mercado y Oudhof (2017) señalan que es conveniente que "el joven cuente con contención, cauce, rumbo y sentido a su natural, frágil e impetuosa forma de ser" (p. 23). Dado que en la juventud, de acuerdo con García (1994), la dimensión predominante es el factor psicosocial, el motor es el motivo, en tanto que la meta es el logro del autodesarrollo. Este último se encuentra asociado a la postura de la persona ante la vida, la que hace posible configurar y asumir una sana actitud frente al propio devenir, en el cual se encuentran inmersas las motivaciones existenciales.

\section{Motivaciones eXISTEnCIAles}

En el existencialismo tienen lugar las interpretaciones personales y subjetivas sobre la vida, las experiencias interiores, los sentimientos, el libre albedrío y la capacidad de elección; asimismo, la individualidad y la responsabilidad personal se tornan el camino más viable para entender el significado de la existencia humana. El análisis existencial plantea que la existencia es un modo específico del ser humano, cuya característica de fondo no es ser fáctico, sino facultativo.

Al respecto, Pareja (2006) menciona que "la diferencia entre el ser fáctico y el ser facultativo reside en que el ser humano siempre puede llegar a ser de-otro-modo y no tiene que ser siempre así, como sucede en la facticidad determinística" (p. 266), ya que es un ser humano condicionado, mas no determinado. El hombre, como ser facultativo, único, irrepetible, libre, responsable, capaz de autodistanciarse y autotrascender, puede enfrentar y valorar los avatares de su vida como una tarea individual que no puede ser ejecutada por ningún otro, ya que solo la persona, en su unicidad e individualidad, tiene los argumentos para ello.

Las motivaciones existenciales son piedras angulares que coadyuvan a hacer posible lo anterior, dado que en el devenir humano se enfrentan situaciones o acontecimientos que suelen tocar la propia vulnerabilidad, sobre todo cuando están acompañados por matices de quebranto como el miedo, el sufrimiento, la angustia; o bien por crisis, cuestionamientos, conflictos o actos que emergen de aquello que no está en las propias manos modificar o cambiar.

Längle (2003) describe las motivaciones existenciales como "la capacidad procesual orientativa de la voluntad constitutiva, por la cual la persona percibe y reconoce algo en su valer integrándolo a su realidad interior y abriéndose libremente a un contexto de sentido" (p. 50). Implican condiciones estructurales de la existencia humana y pueden ser reconocidas a partir de cualquier otra motivación: convicciones, sentimientos y valores, decisiones y significados, $\mathrm{y}$ todo lo que conlleve un dinamismo orientado al autorreconocimiento interno. Son basamento de la existencia humana y son relevantes en todos los ámbitos en donde se establece una relación (Längle, 2004). Emergen de una serie de cuestionamientos 
y condiciones existenciales que elabora la persona sobre su estar en el mundo y que le permiten tener en cuenta su proceso de desarrollo, es decir, si está encaminado a posibilitar o no lo mejor de su ser para dar y darse a sí mismo y a los otros.

Längle (2009) plantea que hay cuatro ámbitos que el ser humano no puede eludir y que tendrá que confrontar toda la vida y en todas las direcciones de su existencia, ya sea en lo cotidiano, en el curso de la vida, en los aspectos límite y en las más difíciles situaciones, ya que ampliarán su horizonte como persona. Son las siguientes:

- El mundo, con sus exigencias y posibilidades.

- La propia vida - la propia naturaleza, con su vitalidad.

- El propio ser persona - el ser uno mismo, la legitimidad, la no intercambiabilidad.

- El futuro, ligado a la exigencia de actuar, de involucrarse activamente en los contextos en que se está y en los que se puede crear. (p. 13)

Si el ser humano puede entrar en un intercambio dialógico con lo interno y externo que está ante él, entonces le será posible dar un fundamento a su propio existir, vivir más profundamente, y mediante la conformación de sí y del mundo tomar una postura que lo lleve al desarrollo de su ser. Solo con el reconocimiento del valor intrínseco de lo que está enfrente a cada momento y en la aceptación del valor propio podrá llegar a la plenitud de su existir.

De esta manera, la relación con el mundo, con la vida, consigo mismo y con el futuro corresponden en el análisis existencial a las categorías fundamentales de la existencia. De ser percibidas en profundidad y vividas adecuadamente, estas se tornan en los elementos constitutivos de una existencia plena. Ahí tiene lugar "la provocación necesaria para responder a todo lo que confronta, desafía y que compromete. Ser provocado significa ser llamado. Esta provocación es el punto de partida para cualquier motivación [...] desde el existencialismo, motivación significa involucramiento de la persona" (Längle, 2008, p. 50). En este sentido, el autor mencionado delinea sus cuatro motivaciones fundamentales de la existencia, las cuales se documentan a continuación:

1. El mundo: relacionarse con condiciones y posibilidades

Esta motivación radica en el hecho de que se está en el mundo, y para ser en el mundo se requiere protección, espacio y apoyo; es decir, vivencias de protección interna y externa, espacio propio y sostén para poder hacer frente a las dificultades; con base en ello, se determina la confianza fundamental. Sin embargo, no es suficiente con encontrar protección, espacio y sostén, también se necesita tomar posesión de estas condiciones, decidirse a su favor; aceptar lo positivo y soportar lo negativo. Por tanto, aceptar significa estar listo para ocupar el espacio, contar con el sostén y confiar en la protección, lo cual implica estar ahí y no tener que huir. Soportar es tener la fuerza necesaria para persistir, a pesar de lo difícil, amenazante o inalterable y asumir lo que no se puede cambiar. Ante ello, a la persona 
solo le resta rendirse desde la aceptación; si no logra plantarse desde la aceptación o bien desde el hecho de soportar lo que la existencia le presenta, vivirá inmersa en la intranquilidad, el miedo y la angustia.

2. La vida: relacionarse con vínculos y emociones

Estar en el mundo no es suficiente, ya que se puede estar, mas no ser. Estar vivo significa llorar y reír, sentir alegría y sufrimiento, tener que vivir las cosas placenteras o las que no lo son tanto, ser afortunado o no serlo, y experimentar el valor y la falta de él. Para compenetrarse con la vida, serequiere de tres condiciones: tener relaciones interpersonales, darse tiempo para uno mismo y experimentar cercanía emocional para conectarse con los valores. Así, la persona podrá encontrar armonía con el mundo y consigo misma, además de sentir la plenitud de la vida. Estas experiencias constituyen la valía fundamental, el más profundo sentimiento hacia el valor de la existencia. Por ello, en su ausencia emergerá la nostalgia, la cual dará pie a la frialdad para finalmente desembocar en una depresión.

3. Ser persona: armonizar consigo mismo y con la otredad

La persona necesita vivir además como un ser separado, mas no indiferente ni aislado. Para lograr esto, es necesario vivenciar hacia sí misma tres aspectos: atención, justicia y consideración. Si estas vivencias faltan, en su lugar se presentará la soledad, la histeria, y la vergüenza, si estas cualidades tienen cabida en la vida de la persona, se encontrará así misma, será autentica, podrá contactar con su consuelo interno, favorecerá el respeto por sí misma. La suma de tales experiencias, por tanto, construye el valor propio, la autoestima. Esta es necesaria para delimitarse, tomar postura y rechazar todo aquello que no corresponda con su mismidad.

4. El sentido: conectarse con el futuro y contextos más amplios

Si la persona tiene la capacidad para estar en el mundo, amar la vida y encontrarse a sí misma, entonces podrá reconocerse como un ser trascendente que sale de sí mismo para contactarse plenamente con el otro. Esto significa que en el contacto humano con los otros podrá ir más allá, al dar lo mejor de sí y retomar como tareas existenciales tanto el amor como el servicio. Längle (2008) señala que sin esta autotrascendencia está condenada a una existencia trivial y solitaria; matizada, en palabras de Frankl (2001), por un sentimiento de vacío, frustración e incluso desesperación. Esto con frecuencia puede resultar en conductas adictivas, agresivas o bien en tendencias suicidas. En cambio, si la persona cuenta con una actitud de apertura y puede manejar las demandas de la vida cotidiana, entonces estará posibilitada para descubrir el sentido de su propia existencia.

Por tanto, se puede estar en concordancia con la situación y examinar si lo que se hace es realmente un bien común. De esta manera, si la persona actúa en consecuencia, su existencia será plena, se constituirá en un ser facultativo.

Ser uno mismo conlleva el ejercicio de estas cuatro motivaciones existenciales, las 
cuales implican una interacción interpersonal e intrapersonal de contenidos y valores que coadyuven a un proceso armónico entre lo que es propio del hombre y lo que le es externo, para que no pierda lo que le da unidad y a la vez le permite ser dialogal, es decir, mantenerse en relación con el mundo, con la otredad. Así, la motivación es ese flujo que se establece entre la persona y su mundo, lo cual no es inseparable. Desde la postura del análisis existencial, vivir implica la oportunidad de colocarse frente a la existencia en una posición de apertura, enfrentar los avatares que se puedan presentar a partir de la confianza y el valor fundamental, el autovalor y el sentido de vida, factores que integran el Test de las Motivaciones Existenciales. Por ello, el objetivo de este estudio se centra en obtener las propiedades psicométricas de dicho instrumento.

\section{Método}

\section{Participantes}

Se trabajó con una muestra no probabilística de tipo accidental conformada por 300 adultos jóvenes. Para ello, se consideraron los siguientes criterios de inclusión: edad comprendida entre los 18 y 25 años, que supieran leer y escribir, con escolaridad mínima de secundaria (a fin de que pudieran comprender las preguntas del instrumento) y residencia en la zona metropolitana del Valle de Toluca.

\section{Instrumento}

El Test de las Motivaciones Existenciales (Test/Zur/Existentielle/Motivation) fue construido por Alfried Längle con la ayuda de Petra Eckhard en el 2000. Se estandarizó en Viena con una muestra al azar compuesta de 1028 participantes de 18 a 69 años. En su versión original contiene 56 reactivos de escala tipo Likert con seis opciones de respuesta, distribuidos en cuatro factores integrados por 14 ítems cada uno de ellos: Confianza fundamental, Valor fundamental, Autoestima y Sentido de vida. Con un total de varianza explicada del 50,15\%, un alfa de Cronbach total de .974 y de $.858, .857$, .773 y .858 para cada una de las escalas.

Gottfried (2015) bajo la supervisión de Längle, aplicó en el 2014, el test a 938 personas en la Argentina en su versión de 66 ítems. El análisis factorial conservó la estructura de la escala original de cuatro factores y los ítems quedaron distribuidos de la siguiente manera: Valor fundamental con 19 ítems, Sentido de vida con 17 ítems, Confianza fundamental con 11 ítems, y Autoestima con 19 ítems. En el estudio de la confiabilidad se tuvo un coeficiente total de alfa de Cronbach de .953; y, para las dimensiones, de .914, .868, .818, y .611, respectivamente.

\section{Procedimiento}

Se contactó a los posibles participantes con la finalidad de aplicarles el Test de la Motivaciones Existenciales, así como la ficha de identificación de las variables demográficas. Se les informó sobre los aspectos generales y el objetivo de la investigación. Se les solicitó su autorización por escrito (firma del consentimiento informado), para lo cual se les garantizó la confidencialidad de sus datos personales y el anonimato. 
Los datos recabados a partir de la aplicación del instrumento fueron ingresados en el paquete estadístico SPSSS versión 23 para su procesamiento estadístico. Se obtuvo la medida de adecuación muestral (KMO), la prueba de esfericidad de Bartlett y el criterio de Kaiser. La validez de constructo se llevó a cabo a partir del análisis factorial exploratorio, con rotación ortogonal tipo Varimax y método de extracción de máxima verosimilitud, considerando como criterios pesos factoriales superiores a 0,40 y autovalor superior a 1 (Hair, Anderson, Tatham y Black, 2004).

El cálculo de fiabilidad se hizo a través del alfa de Cronbach $(\alpha)$, según el criterio de Oviedo y Campo-Arias (2005), el cual establece como valor mínimo aceptable 0,70 ; por debajo de ese valor la consistencia interna de la escala utilizada es baja.
Por su parte, el valor máximo esperado es 0,90 ; por encima de este valor se considera que hay redundancia o duplicación, por lo que usualmente se prefieren valores de alfa entre 0,80 y 0,90 .

\section{Resultados}

El índice de la prueba Káiser, Meyer y Olkin arrojó un valor de .95 , lo cual indicó que la muestra era adecuada para realizar el análisis factorial. Por otra parte, el de la prueba de Esfericidad de Barlett fue de .00, lo que conllevó a establecer que los reactivos se encontraban intercorrelacionados y determinó la pertinencia de llevar a cabo el análisis factorial. A partir del análisis factorial exploratorio, se obtuvieron cuatro factores que explicaron el 50,22\% de la varianza total (ver tabla 1 ).

Tabla 1

Valores eigen, porcentajes de varianza y porcentajes acumulativos para los factores de la Escala de Propósito de Vida para Adultos Mayores

\begin{tabular}{c|c|c|c}
\hline Factor & Valor eigen & $\begin{array}{c}\text { Porcentaje } \\
\text { de varianza }\end{array}$ & $\begin{array}{c}\text { Porcentaje } \\
\text { acumulativo }\end{array}$ \\
\hline 1 & 9,42 & 16,82 & 16,82 \\
2 & 7,63 & 12,83 & 29,65 \\
3 & 6,30 & 11,23 & 40,88 \\
4 & 5,23 & 9,34 & 50,22 \\
\hline
\end{tabular}

El test quedó integrado por 52 reactivos debido a que se eliminaron los ítems 17,18 , 19 y 26, que se distribuyeron de la siguiente manera: El primer factor, Sentido de vida, quedó integrado por 17 ítems; el segundo,
Autovaloración, agrupó 15; el tercero, Valor fundamental, quedó conformado por 12; y el cuarto, Confianza fundamental, por 8 (ver tabla 2). 
Tabla 2

Resumen de reactivos y cargas factoriales para una solución ortogonal varimax de cuatro factores para el Test de Motivaciones Fundamentales en adultos jóvenes

\begin{tabular}{|c|c|c|c|c|c|}
\hline & & \multicolumn{4}{|c|}{ Factores } \\
\hline & & 1 & 2 & 3 & 4 \\
\hline & & SV & AV & VF & $\mathrm{CF}$ \\
\hline 1. & He encontrado mi lugar en la vida. & & & & .527 \\
\hline 2 & Acepto con buena disposición las circunstancias de mi vida. & & & & .550 \\
\hline 3. & Me procuro el espacio que necesito para mi vida. & & & & .586 \\
\hline 4. & Siento que no estoy vivo/a. & & & .579 & \\
\hline 5. & Estoy contento conmigo mismo/a. & & & & 679 \\
\hline 6. & Me siento capacitado/a para las exigencias de la vida. & & & & .615 \\
\hline 7. & Soy vergonzoso/a. & & .448 & & \\
\hline 8. & Tengo respeto por mí mismo/a. & & & & .658 \\
\hline 9. & Estoy conforme con ser como soy. & & & & .583 \\
\hline 10. & Nada me da sostén en la vida. & & & .657 & \\
\hline 11. & Estoy a mi favor. & .489 & & & \\
\hline 12. & Me siento bien en mi cuerpo. & & & & .533 \\
\hline 13. & Me siento desubicado/a. & & .480 & & \\
\hline 14. & Me siento solo/a. & & .441 & & \\
\hline 15. & Me siento contento/a. & .470 & & & \\
\hline 16. & Me gustaría desaparecer de la faz de la Tierra. & & & .684 & \\
\hline 20. & Soy una persona triste. & & & .474 & \\
\hline 21. & En realidad, hubiese querido no haber hecho lo que hice. & & .457 & & \\
\hline 22. & Siento que tengo suficiente espacio para vivir. & .605 & & & \\
\hline 23. & Tengo la vivencia de tener mucha alegría. & .671 & & & \\
\hline 24. & Me siento inseguro/a. & & .677 & & \\
\hline 25. & Me cuesta aceptarme a mí mismo/a. & & .543 & & \\
\hline 27. & Mi vida no tiene sentido, la siento vacía. & .412 & & & \\
\hline 28. & Siento que les gusto a los demás. & .460 & & & \\
\hline 29. & Me aprecio, me valoro por mi rendimiento y eficiencia. & .572 & & & \\
\hline 30. & Experimento la vida como una carga. & & & .574 & \\
\hline 31. & En el fondo rechazo estar vivo/a. & & & .764 & \\
\hline 32. & La inseguridad en mi vida me angustia. & & .532 & & \\
\hline 33. & Me siento agobiado/a por la vida. & & & .479 & \\
\hline 34. & Hago lo que quiero hacer. & .609 & & & \\
\hline 35. & Tengo una buena disposición hacia mí mismo/a. & .600 & & & \\
\hline
\end{tabular}


(continuación)

36. Siento que mi entorno respeta mis deseos y decisiones.

.641

37. Por mi miedo al fracaso no empiezo muchas cosas.

.658

38. Siento que los demás tienen más razón que yo.

39. Estoy convencido de que es bueno estar en este mundo.

.458 .

40. La mayor parte de lo que he hecho ha sido inútil.

.401

41. Siento que tengo apoyo en mi vida.

.626

42. Me gusto tal y como soy.

.591

43. En mi opinión rindo demasiado poco.

44. Mi actuar me hace sentir vivo.

.693

45. Pocas veces sé lo que quiero.

46. Amo la vida.

47. Considero que me hace falta realizar mis planes.

48. Experimento con frecuencia que soy muy bueno para algo. .600

49. Siento que en el fondo es bueno que yo no exista.

50. Soy exitoso/a.

.693

51. Me gusta vivir.

52. Me es difícil saber para que estoy en la vida.

53. Recibo poco afecto de los demás.

54. Soy una persona tímida.

55. Sé para qué hago algo.

56. Encamino mi vida con sentido. .650

Nota. Se eliminaron los ítems 17, 18, 19 y 26.

En cuanto a la fiabilidad del test y cada uno de los factores que lo integran, esta se calculó mediante el coeficiente alfa de
Cronbach. Los resultados se presentan en la tabla 3: 
Tabla 3

\section{Coeficientes de fiabilidad}

\begin{tabular}{lcl}
\hline Factor & & Alfa de Cronbach (a) \\
\hline Sentido de vida & $(\mathrm{n}=17)$ & .94 \\
Autovaloración & $(\mathrm{n}=15)$ & .88 \\
Valor fundamental & $(\mathrm{n}=12)$ & .89 \\
Confianza fundamental & $(\mathrm{n}=8)$ & .86 \\
Motivaciones existenciales $(\mathrm{n}=52)$ & .96
\end{tabular}

\section{Discusión}

Los puntajes obtenidos tanto en el índice de la prueba Káiser, Meyer y Olkin como en la prueba de Esfericidad de Bartlett indicaron que la muestra era adecuada para realizar el análisis factorial. Asimismo, se determinó que los reactivos se encontraban intercorrelacionados, por lo que se pudo llevar a cabo el análisis factorial (Pere y Anguiano, 2010).

A partir de los estudios previos en cuanto a la estructura del Test de Motivaciones Existenciales, efectuados por Längle y Eckhard (2000) y Gottfried (2015), y del fundamento teórico de dicho instrumento, se utilizó un análisis factorial de tipo exploratorio con la finalidad de confirmar la estructura original del instrumento de cuatro factores. El análisis de componentes principales de tipo ortogonal varimax permitió extraer de manera sucesiva cada uno de los factores.

El análisis factorial exploratorio identificó cuatro factores claramente independientes, en los que se integraron 52 reactivos de los 56 que componen el instrumento original. Los cuatro que se eliminaron presentaban una carga factorial menor de 0,40 . Los valores eigen obtenidos, de acuerdo con los criterios referidos por Zamora, Monroy y Chávez (2009), garantizan que los factores expliquen la varianza total obtenida, de $50,22 \%$, que en este estudio fue superior a la obtenida por Gottfried (2015) y explicó $42,86 \%$ de la variabilidad de la varianza total. De acuerdo con el porcentaje, se tuvo que el primer factor explicó la mayor varianza común, y los otros tres la varianza residual.

Los resultados mostraron una adecuada estructura de correlación, es decir, que los factores explicaron la asociación entre cada uno de los reactivos. En cuanto a la carga factorial, esta permitió identificar los reactivos más representativos de cada uno de los factores. La solución rotada permitió clarificar la estructura de las cargas factoriales con la finalidad de facilitar su interpretación (Zamora et al., 2009).

La evidencia empírica a partir de los datos obtenidos permite establecer que el ajuste estadístico de los datos al modelo 
teórico es satisfactorio, por lo que la estructura de cuatro factores para la población de estudio es adecuada (Brown, 2006). Por otra parte, la fiabilidad calculada por el alfa de Cronbach fue mayor de .80 para cada uno de los factores y de .96 para el total de la prueba, lo cual indica una alta confiabilidad. Lo anterior es compatible con los resultados del estudio de Gottfried (2015), en el que se reporta una alta y aceptable confiabilidad.

\section{CONCLUSIONES}

A partir de los análisis estadísticos efectuados, se puede considerar que el test cuenta con las propiedades psicométricas adecuadas para la medición de las motivaciones existenciales en adultos jóvenes.

Se conservó la estructura factorial propuesta en el instrumento original, de cuatro factores, a partir del análisis factorial exploratorio. Por ello, el test muestra evidencias de validez basadas en la estructura interna.

La consistencia interna de la escala, a partir de los valores de las alfas de Cronbach, por factor y total indican que las puntuaciones derivadas del instrumento presentan alta fiabilidad.

\section{ReferenCIAS}

Berger, K. S. (2009). Psicología del desarrollo. Adultez y vejez (7.a ed.) Madrid: Médica Panamericana.

Brown, T. A. (2006). Confirmatory Factor Analysis for Applied Research. Nueva York: The Guilford Press.

Frankl, V. (2001). Ante el vacío existencial. Hacia una humanización de la psicoterapia (8. ${ }^{\mathrm{a}}$ ed.). Barcelona: Herder.

García, C. (1994). Sobre la rehumanización de la psicología y el proceso de maduración noética. En E. Lukas y C. García (Eds.), De la vida fugaz (pp. 59-145). Buenos Aires: Almagesto.

Gottfried, A. (2015). Manual del Test de las Motivaciones Existenciales. Argentina: GLE.

Hair, J., Anderson, R, Tatham, R., y Black, W. (2004). Análisis Multivariante. Ciudad de México: Prentice-Hall.

Längle, A. (2003). The Art of Involving the Person. En European Psychotterapy, 4(1), 47-58.

Längle, A. (2004). The Search for Meaning in Life and the Existential Fundamental Motivations. The International Journal of Existential Psychology' and Psychotherapy, 1(1), 28-37.

Längle, A. (2008). Vivir con sentido. Aplicación práctica de la logoterapia. Buenos Aires: Lumen.

Längle, A., y Eckhardt, P. (2000). Test zur Existentiellen Motivation (TEM). Wien: GLE-Verlag. 
Mercado, A., y Oudhof, H. (2017). Adolescencia de la inmanencia a la autotrascendencia. En E. Robles, H. Oudhof y A. Mercado (Eds.), Adolescencia y juventud: procesos de construcción personal y social. México: Colofón.

Instituto Nacional de Estadística y Geografía. (2016). Encuesta intercensal EIC 2015. Base de datos. México. Recuperado de http:www.inegi.org.mex/contenidos/programas/ intercensal/2015/doc/oec_2015_presentacion.pdf

Instituto Nacional de Estadística y Geografía. (2018). Encuesta nacional de ocupación y empleo ENOE. Recuperado de http://www.inegi.org.mx/contenidos/saladeprensa/ boletines2018/enoe/enoe_ie2018_11.pdf

Organización de las Naciones Unidas. (2016). Juventud. Recuperado de www.un.org/es/ globalissues/youth

Oviedo, H. C., y Campo-Arias, A. (2005). Aproximación al uso del coeficiente alfa de Cronbach. Revista Colombiana de Psiquiatría, 34(4), 572-580.

Pareja, G. (2006). Viktor E. Frankl: Comunicación y resistencia. Buenos Aires: San Pablo.

Pere, F., y Anguiano, C. (2010). El análisis factorial como técnica de investigación en psicología. Papeles de Psicólogo, 1(31). Recuperado de http://www.papelesdelpsicologo.es/

Rice, P., (2006). Desarrollo humano. Estudio del ciclo vital. México: Prentice Hall.

Trucco, D, y Ullman, H. (Eds.). (2015). Juventud: realidades y retos para un desarrollo con igualdad. Santiago de Chile: Comisión Económica para América Latina y el Caribe.

Zamora, S., Monroy, L., y Chávez, C. (2009). Análisis factorial: una técnica para evaluar la dimensionalidad de las pruebas. Ciudad de México: Ceneval. 